\title{
Phylogenetic analysis of rabbit haemorrhagic disease virus (RHDV) strains isolated in Poland
}

\author{
Andrzej Fitzner ${ }^{1} \cdot$ Wieslaw Niedbalski $^{1}$
}

Received: 15 March 2017 / Accepted: 13 June 2017 / Published online: 12 July 2017

(C) The Author(s) 2017. This article is an open access publication

\begin{abstract}
The aim of this study was to characterise the nucleotide and amino acid sequence of complete genomes $(7.5 \mathrm{~kb})$ from RHDV strains isolated in Poland and estimate the genetic variability in different elements of the viral RNA. In addition, the sequence of Polish RHDV isolates isolated from 1988-2015 was compared with the sequences of other European RHDV, including the RHDVa and RHDV2/RHDVb subtypes. The complete sequence was developed by the compilation of partial nucleotide sequences. This sequence consisted of approximately 7428 nucleotides. For comparison of nucleotide sequences and the development of phylogenetic trees of Polish RHDV isolates and reference RHDV strains representing the main phylogenetic groups of classical RHDV, RHDVa and RHDV2 as well as the non-pathogenic rabbit lagovirus RCV, the BLAST software with blastn and MEGA6 with neighbour-joining method was applied. The complete nucleotide sequence of Polish isolates of RHDV has also been entered into GenBank. For comparative analysis, nineteen complete sequences representing the main RHDV genetic types available in GenBank were used. The results of phylogenetic analysis of Polish RHDV strains reveals the presence of three classical RHDV genogroups (G2, G4 and G5) and an RHDVa variant (G6). The oldest RHDV isolates (KGM 1988, PD 1989 and MAL 1994) belong to genogroup G2. It can be assumed that the elimination of these strains from the environment probably occurred at the turn of 1994 and 1995. Genogroup G2 was replaced by the phylogenetically younger BLA 1994 and OPO 2004 strains
\end{abstract}

Andrzej Fitzner

andrzej.fitzner@piwzp.pl

1 Department of Foot and Mouth Disease, National Veterinary Research Institute, Wodna 7, 98-220 Zduńska Wola, Poland from genogroup $\mathrm{G} 4$, which probably originated from the G3 lineage, represented by the Italian strains BS89. The last representatives of classical RHDV in Poland are isolates GSK 1988 and ZD0 2000 from genogroup G5. A single clade contains the Polish RHDV strains from 2004-2015 (GRZ 2004, KRY 2004, L145 2004, W147 2005, SKO 2013, GLE 2013, RED1 2013, STR 2012, STR2 2013, STR 2014, BIE 2015) identified as RHDVa, which clustered into genogroup G6, as represented by the RHDV strain Triptis 1996. All recent isolated RHDV isolates belong exclusively to RHDVa and no RHDV2 was diagnosed in Poland.

Keywords Rabbit haemorrhagic disease virus - Complete nucleotide sequence $\cdot$ Phylogenetic analysis

Rabbit haemorrhagic disease (RHD) is a highly contagious and often lethal viral infection of wild and domestic rabbits (Oryctolagus cuniculus), which first emerged in China in 1984 [19]. Over the next years RHD spread throughout the world affecting many countries, mainly in Asia and Europe but also in Africa, Central and North America, Australia and New Zealand [1]. The etiological agent, rabbit haemorrhagic disease virus (RHDV) is an icosahedral, non-enveloped, single-stranded positive-sense RNA virus of approximately 7437 nucleotides with two open reading frames (ORFs). The longer ORF1 (7034 nucleotides) encodes seven non-structural viral proteins p16, p23, p37 (helicase), p29, virus protein-genome linked (VPg), p15 (3C-like proteinase), p58 (RdRp) and a structural capsid proteinviral protein (VP60). The shorter ORF2 (353 nucleotides) encodes viral protein VP10 which, so far, has an unknown function [25]. It is well known that RNA viruses are characterized by their great potential for variability; however, RHDV strains isolated at the beginning of epizootic were 
conservative in terms of antigenic and genetic features regardless of the time and place of isolation. The first significant changes in the properties of the virus related to the lack of haemagglutination capacity in some RHDV strains [14]. In 1996, the occurrence of a new RHDV virus - the RHDVa variant - was reported in Italy and Germany [5, 27] and a non-pathogenic calicivirus (RCV) related to was also diagnosed in rabbits [4]. In 2010, a new RHDV subtype, designated as RHDV2, genetically and antigenically different from the classical RHDV and RHDVa variant, spread in Europe $[7,17,18]$. To explain the origin and the emergence of the new RHDV variant two hypotheses were proposed: 1) evolution from pre-existing non-pathogenic viruses circulating in Europe, and 2) a species jump [1]. On the basis of phylogenetic relationships, the pathogenic RHDV strains have been divided into three distinct groups: classical RHDV with the genogroups G1-G5 [26], the antigenic variant RHDVa (genogroup G6) [14], and the new subtype RHDV2 [16]. Distribution of RHDV into several genetic groups was developed on the basis of a similar time of virus isolation, regardless of the geographical distribution of disease [16]. The oldest RHDV isolates belong to the groups G1-G3. The representative of G1 group is RHDV strain AST89 isolated in Spain in 1989. The German strain FRG89 - the first sequenced RHDV and the Czech strain V351, introduced in 1995 in Australia and New Zealand to control the populations of wild rabbits, are representatives of the genetic group G2. The Italian RHDV strain BS89 isolated in 1989 and the German vaccine strain Meiningen are clustered into group G3. Genogroups G4 and G5 comprise many European RHDV from the 90s onwards, as well as the beginning of 21st century. All known strains of RHDVa variant are clustered into genogroup G6 [18].

In Poland, the first cases of RHDV were confirmed in 1988 [13]. In the following years, the disease spread rapidly and caused significant losses in rabbitries, regardless of race and type of breeding rabbits. In 1994, a non-haemagglutinating RHDV strain, defined as BLA, after the place of isolation (Blaszki village) was recognized, and then in middle of the first decade of the 21st century, RHDV strains exhibiting features similar to the RHDVa variant were isolated in Poland [6, 9]. To date, no RHD outbreaks caused by the RHDV2 subtype were confirmed in Poland. The main reason for this could be a specific structure of lagomorphs species living in Poland, with the presence of only domestic rabbits (Oryctolagus cuniculus) and brown hares (Lepus europaeus). Wild rabbits living freely, or in captivity, as well as other related species of rabbits (eg. Sylvilagus floridanus) do not occur naturally in the environment. Another important aspect is the structure of the rabbit farm with a dominance of mixed-breed rabbits reared in smallscale farms and a relatively few number of industrial farms. The current epidemiological situation regarding RHD and serological status of rabbits has recently been described [12].

The first phylogenetic analysis of Polish RHDV strains collected between 1988 and 2000 (SGM 1988, KGM 1988, LUB 1988, PD 1989, MAL 1994, BLA 1994, GSK 1988, ZD0 2000), was performed in our laboratory in 2003 [10]. These studies were based on the nucleotide sequences encoding the N-terminal fragment of VP60 and a fragment of the p29 gene sequence. Nucleotide and amino-acid sequences from RHDV strains revealed a high genetic identity of isolates and two genetic groups showing temporal similarities were determined. Subsequently, to estimate the genetic variability of Polish RHDV strains and to confirm the presence of the genetic variant RHDVa, the partial nucleotide sequence of the capsid protein gene $V P 60$, including two highly variable regions $\mathrm{C}$ and $\mathrm{E}$, was determined [11]. Phylogenetic analysis of 15 RHDV strains isolated over a period of 18 years revealed the presence of three genetic groups. The oldest RHDV showed a very high similarity at the amino acid level (98-99\%) to the German FRG89 reference strain and to most European viruses isolated in the same period, and also to the Chinese isolate from 1984. The non-haemagglutinating (HA-negative) strains clustered into a second subgroup presenting an intermediate degree of genetic variability (about 3\%) in the VP60 gene fragment. The most genetically variable strains (6-7\%) clustered in the RHDVa subtype [11].

The aim of this study was the characterisation of the nucleotide and amino acid sequences of the complete genomes $(7.5 \mathrm{~kb})$ of RHDV strains isolated in Poland and an estimation of the genetic variability in different elements of the viral RNA. In addition, the nucleotide sequence of Polish RHDV isolates from 1988-2015 were compared to sequences of other European RHD viruses, including the RHDVa and RHDV2/RHDVb subtypes. Total RNA was extracted from rabbit liver homogenates as described previously [11]. cDNA was synthesized using oligo dT15 and AMV reverse transcription enzyme (Promega). Thirty one pairs of specific oligonucleotide primers targeting different fragments of the RHDV genome were used in alternative combinations to amplify and sequence 33 RHDV RNA fragments. PCR products were analysed on a $1.5 \%$ agarose gel, stained with ethidium bromide and visualized with ImageStore documentation system (UVP). Specific PCR products of 434-856 bp from eighteen Polish RHDV strains collected between 1988 and 2015 were obtained (Table 1). The PCR products were gel-extracted and directly sequenced using $\mathrm{ABI}$ Prism BigDye ${ }^{\mathrm{TM}}$ terminator v3.1Cycle sequencing kit and ABI3773x1 DNA sequencer (Applied Biosystems). The sequence of the full RHDV genome was determined after analysis of partial sequences available in the form of text file (txt) and fluorograms (file. abi) and by the contiguous compilation of these partial 
Table 1 Nucleotide similarity (\%) between sequences of 18 Polish RHDV strains as well as representatives from the G1-G6 genogroups, RHDV2, RCV-like (RCV-A1, MRCV) and RHDV FRG89 strains (M67473)/G2

\begin{tabular}{|c|c|c|c|c|c|c|c|c|}
\hline & \multirow{3}{*}{$\begin{array}{l}\text { RHDV strain } \\
\text { (year of } \\
\text { isolation) }\end{array}$} & \multirow[t]{3}{*}{ Acc. no. } & \multicolumn{4}{|l|}{ ORF1 } & \multirow{2}{*}{$\begin{array}{l}\text { ORF2 } \\
\text { VP10 }\end{array}$} & \multirow[t]{2}{*}{$\mathrm{CG}$} \\
\hline & & & $\begin{array}{l}\text { NS1-NS7 } \\
\text { (average) }\end{array}$ & $\begin{array}{l}\text { VP60 E I sub- } \\
\text { region }\end{array}$ & $\begin{array}{l}\text { VP60 E II sub- } \\
\text { region }\end{array}$ & VP60 (average) & & \\
\hline & & & $10-5304$ & $6334-6414$ & $6496-6606$ & $5305-7041$ & $7025-7378$ & $10-7437$ \\
\hline \multirow{18}{*}{$\begin{array}{l}\text { Polish RHDV } \\
\text { strains }\end{array}$} & KGM (1988) & KP144790 & 99 & 99 & 100 & 99 & 99 & 99 \\
\hline & PD (1989) & KP144789 & 99 & 99 & 100 & 99 & 99 & 99 \\
\hline & MAL (1994) & KU882093 & 99 & 99 & 100 & 98 & 99 & 99 \\
\hline & BLA (1994) & KP144792 & 94 & 91 & 97 & 96 & 96 & 95 \\
\hline & GSK (1998) & KU882092 & 94 & 95 & 95 & 96 & 95 & 94 \\
\hline & ZD0 (2000) & KU882094 & 94 & 94 & 94 & 95 & 96 & 94 \\
\hline & OPO (2004) & KU882094 & 94 & 90 & 97 & 96 & 96 & 94 \\
\hline & GRZ (2004) & KP144791 & 89 & 85 & 90 & 92 & 96 & 90 \\
\hline & KRY (2004) & KY319033 & 89 & 87 & 91 & 93 & 97 & 90 \\
\hline & L145 (2004) & KY679902 & 89 & 85 & 90 & 92 & 96 & 90 \\
\hline & W147 (2005) & KY319035 & 89 & 85 & 90 & 92 & 96 & 90 \\
\hline & STR (2012) & KF677011 & 88 & 84 & 92 & 92 & 95 & 90 \\
\hline & STR2 (2013) & KY679904 & 88 & 85 & 90 & 92 & 95 & 89 \\
\hline & GLE (2013) & KY319032 & 88 & 85 & 92 & 92 & 95 & 90 \\
\hline & RED1 (2013) & KY679903 & 88 & 84 & 89 & 92 & 97 & 90 \\
\hline & SKO (2013) & KY319034 & 88 & 85 & 91 & 92 & 95 & 90 \\
\hline & STR (2014) & KY679905 & 88 & 84 & 91 & 92 & 95 & 91 \\
\hline & BIE (2015) & KY319031 & 88 & 84 & 91 & 92 & 95 & 90 \\
\hline \multirow{18}{*}{$\begin{array}{l}\text { Representatives } \\
\text { of RHDV and } \\
\text { RCV types }\end{array}$} & V351 (1987) & U54983 & 99 & 99 & 99 & 99 & 99 & 99 \\
\hline & BS (1989) & X87607 & 94 & 94 & 95 & 96 & 96 & 95 \\
\hline & SD (1989) & Z29514 & 94 & 96 & 95 & 95 & 95 & 94 \\
\hline & AST (1989) & Z49271 & 94 & 96 & 95 & 96 & 96 & 95 \\
\hline & $\begin{array}{l}\text { Eisenhuttenstadt } \\
\text { (1989) }\end{array}$ & EF558578 & 94 & 96 & 94 & 95 & 96 & 95 \\
\hline & $\begin{array}{l}\text { Meiningen } \\
\text { (1993) }\end{array}$ & EF558577 & 94 & 94 & 95 & 96 & 96 & 94 \\
\hline & Jena (1993) & EF558576 & 94 & 94 & 95 & 96 & 96 & 94 \\
\hline & $\begin{array}{c}\text { Frankfurt5 } \\
\text { (1996) }\end{array}$ & EF558573 & 94 & 91 & 95 & 96 & 95 & 94 \\
\hline & $\begin{array}{l}\text { Frankfurt12 } \\
\text { (1996) }\end{array}$ & EF558572 & 94 & 91 & 95 & 96 & 95 & 94 \\
\hline & Triptis (1996) & EF558583 & 89 & 87 & 90 & 93 & 97 & 90 \\
\hline & $\begin{array}{l}\text { Hartmannsdorf } \\
\text { (1996) }\end{array}$ & EF558586 & 89 & 85 & 91 & 94 & 97 & 90 \\
\hline & Rossi (2002) & EF558584 & 89 & 95 & 96 & 93 & 96 & 90 \\
\hline & NZ61 (2002) & EF558580 & 97 & 96 & 98 & 98 & 98 & 97 \\
\hline & N11 (2011) & KM878681 & 86 & 78 & & 82 & 86 & 85 \\
\hline & CBVal16 (2012) & KM979445 & 86 & 80 & 80 & 81 & 85 & 85 \\
\hline & Algarve1 (2013) & KF442961 & 77 & 78 & 80 & 78 & 85 & 78 \\
\hline & MRCV (2001) & GQ166866 & 78 & - & - & 84 & 88 & 80 \\
\hline & RCV-A1 (2007) & EU871528 & 78 & - & - & 80 & 85 & 78 \\
\hline
\end{tabular}

Similarities are shown for the nonstructural and structural parts of ORF1 and ORF2 of the RHDV genome, as calculated by BLAST (Altschul et al. 1990)

nucleotide sequences. This sequence consists of approximately 7428 nucleotides however the first nine nucleotides from the non-translated 5'UTR region of the RHDV genome were not included in the sequence. Identification of this fragment was not always possible because it contains the nucleotide primer sequence. Taking into consideration 
that this section of the viral RNA genome is relatively stable, it can be assumed that the sequence of the Polish RHDV isolates in this fragment is $100 \%$ identical to the sequence of the reference control strain FRG89 and therefore the nucleotide forward primer [22]. Moreover, according to the structure of the RHDV genome [23, 25], the sequence of the first 16 nucleotides from the 5'UTR region is duplicated in genome fragment 5296-5311, i.e. immediately preceding the VP60 gene [15, 24]. This is why, the position numbering of nucleotides and appropriate ORFs in the complete nucleotide sequence of Polish RHDV isolates has been moved nine positions in relation to the control German strain FRG89. Each RHDV complete sequence contained seven regions of non-structural proteins (NS 1-7), two regions of structural capsid proteins - VP60 and VP10 and a 3'UTR region. For comparison of the nucleotide sequences of the Polish RHDV isolates, reference RHDVs representing the main phylogenetic groups of classical RHDV, RHDVa and RHDV2 as well as the non-pathogenic rabbit lagovirus RCV were examined using BLAST software with blastn [2] and MEGA6 [28] (phylogenetic trees were constructed using the neighbor-joining method). The complete nucleotide sequence of Polish isolates of RHDV has been entered into GenBank. The accession number of the isolates representing the separate genogroups of RHDV, RHDVa, RHDV2 and non-pathogenic virus RCV and RCV-A1 are as follows: FRG 1989 (M67473), AST89 (Z49271), Eisenhuttenstadt 1989 (EF558578), V351 1987 (U54983), BS89 (X87607), SD 1989 (Z29514), Meiningen 1993 (EF558577), Jena 1993 (EF558576), Frankfurt5 1996 (EF558573), Frankfurt12 1996 (EF558572), Triptis 1996 (EF558583), Hartmannsdorf 1996 (EF558586), Rossi 2002 (EF558584), NZ61 (EF558580), N11 (Navarra 2011) (KM878681), CBVal16 (Valpacos 2012) (X96868), Algarve1 2013 (KF442961), RCV-A1 MIC 2007 (EU871528), MRCV 2001 (GQ166866).

The results of the comparison between nucleotide sequences of eighteen Polish RHDV isolates and the reference strain FRG89 are presented in table 1. This German RHDV strain from 1989 was chosen as the reference due to the similar time and geographical location of isolation. Additionally, eighteen complete sequences representing the main RHDV genetic groups from GenBank were compared. The percentage identity of the complete genome (CG), as well as individual genes encoding the non-structural proteins (NSP 1-7), structural capsid protein VP60, E1 and E2 sub-regions of VP60 encoded by ORF1 and the structural capsid protein VP10 encoded by ORF2 are shown. The overall similarity of the complete sequence of Polish isolates of RHDV reflects the similarity of individual fragments within the genome, except the EI sub-region of the capsid protein where some strains are much more differentiated. The relative identity of the genome sequences of these RHDV isolates confirms a similar evolution pattern amongst RHDV strains, and the probable lack of recombinants. The significant process of recombination involving large fragments of the genome has recently been recorded for RHDV2 viruses in the Iberian Peninsula [21]. On the basis of the results obtained for the complete genome sequences (CG, 10-7437), the RHDV isolates were divided into three groups: the first group consisted of classical RHDV strains (99\% sequence identity), and includes the oldest RHDV isolates most similar to RHDV from 90s (KGM, PD and MAL). In the second group were classical RHDVs showing lower genetic similarity (94-95\%). This group consisted of four RHDV isolates from 1994-2004, two of which (BLA 1994 and OPO 2004) are non-haemagglutinating strains that show decreased similarity of $90-91 \%$ in the EI sub-region. The other two isolates were classified in the phylogenetically youngest genogroup of RHDV: ZD0 2000 characterized by its variable reactivity in the haemagglutination assay and GSK 1998 that was positive in this assay. The third group (90-91\% sequence identity) refers to the RHDVa strains from 2004-2015 that demonstrate $84-87 \%$ similarity within the nucleotide sequence of the EI subregion. The nucleotide homology at different regions of the RHDV genome is shown in Table 1. The results of the phylogenetic analysis of the Polish RHDV strains reveals the presence of three classical RHDV genogroups (G2, G4 and G5) as well as the variant RHDVa (G6). Figure 1 presents the dendrogram of the 18 Polish RHDV isolates on the basis of the RHDV complete genome sequence (7.4 $\mathrm{kb}$ ). The oldest RHDV isolates (KGM 1988, PD 1989 and MAL 1994) clustered into genogroup G2. It can be assumed that the elimination of these strains from the environment occurred at the turn of 1994 and 1995. Genogroup G2 was replaced by the phylogenetically younger strains BLA 1994 and OPO 2004 which clustered into the G4 genogroup, which probably originated from the G3 lineage as represented by the Italian strain BS89. It is noteworthy the significant difference in time of isolation (about 10 years) of the BLA and OPO strains that represent a rare non-haemagglutinating subgroup, considered a phenotypic variant [5]. The last representatives of Polish classical RHDV are isolates GSK 1988 and ZD0 2000 from genogroup G5. A single clade contains the Polish RHDV strains from 2004-2015 (GRZ 2004, KRY 2004, L145 2004, W147 2005, SKO 2013, GLE 2013, RED1 2013, STR 2012, STR2 2013, STR 2014, BIE 2015) identified as RHDVa, which clustered into genogroup G6 as represented by RHDV strain Triptis 1996 and Rossi 2002. In this genogroup three smaller subgroups can be selected (Fig. 1). On the basis of epidemiological data and the results of antigenic and genetic analysis, it can be assumed that the RHDVa variant appeared in Poland at 


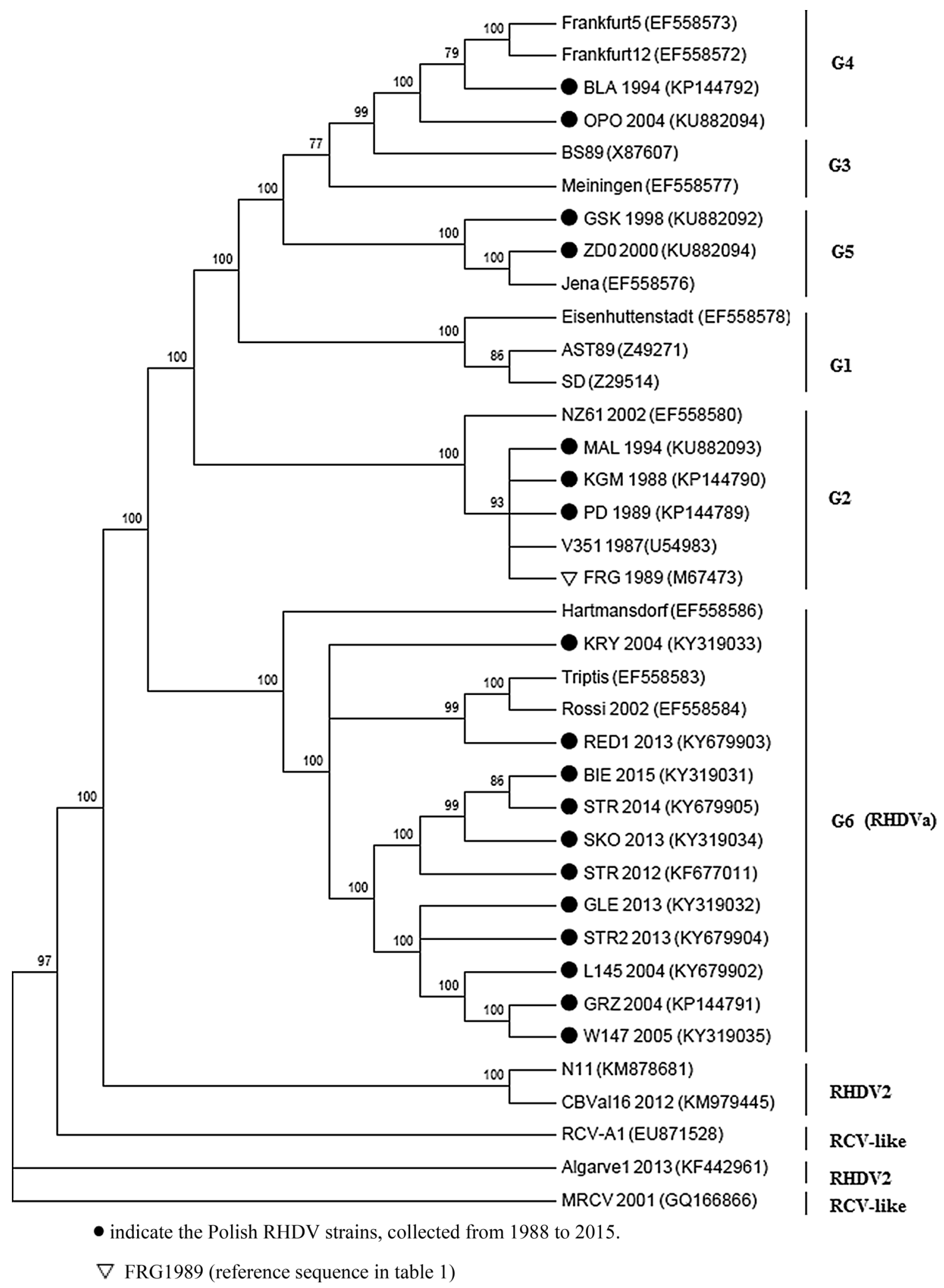

Fig. 1 Neighbor-Joining tree of 37 RHDV strains based on CG sequences. The percentage of replicate trees $(>70)$ in which the associated taxa clustered together in the bootstrap test (1000 replicates) are shown above the branches. Evolutionary analyses were conducted in MEGA6 (Tamura et al. [28]) 
the turn of 2003/2004. In 2004 an epizootic of new RHD outbreaks was observed in Poland, from which haemagglutinating RHDV strains with differing antigen reactions by ELISA assay were isolated [10]. The circulation of RHDV with similar antigenic properties was confirmed repeatedly in 2004-2006. More information about the diagnosis of RHDVa via detection of the VP60 gene using various molecular techniques was presented previously $[6,10]$. Moreover, the antigenic subtype RHDVa was isolated from new RHD outbreaks in Poland in 2012-2016 in the voivodeships podkarpackie, malopolskie and lodzkie (http://www.oie.int/wahis_2/public/wahid.php). The separate clade consisted of RHDV2 strains, which exhibit a greater similarity to the non-haemagglutinating rabbit caliciviruses (Italian RCV, Australian RCV-A1 and American MRCV) [1, 8]. In the last years, the disappearance of classical RHDV strains and the subsequent domination of the RHDVa variant has been observed in Europe, with the exception of the Iberian Peninsula, where until recently the classical RHDV strains from G1 and G5 genogroups were isolated [20]. At present, RHDVa is the dominant virus in Poland; its recognition in RHD outbreaks in 2012-2016 is evidence of continued circulation of this virus in the environment. The spread of RHDV2 in many West European countries and in Scandinavia, Great Britain and Ireland has also been recorded recently $[3,18]$.

In conclusion, in this study we have reported the complete genome sequence of 18 RHDV strains isolated in Poland between 1998 and 2015. On the basis of comparative analysis of their sequences it can be stated that the genome of these viruses is relatively stable, and although no recombination has been demonstrated the presence of this phenomenon cannot be ruled out. At the end of March 2017, eighteen complete genome sequences were entered in GenBank, of which nine have been published and the next are in the process of verification. The results of sequencing of the oldest Polish isolates from 1988-1994 as well as younger strains from 2012-2015 has confirmed the disappearance of classical RHDV in Poland. Accordingly, all recently isolated RHDV strains belong exclusively to RHDVa; however, no RHDV2 has been diagnosed in Poland.

\section{Compliance with ethical standards}

Conflict of interest The authors declare that they have no conflict of interest.

Ethical approval This article does not contain any studies with animals performed by any of the authors.

Funding This study was supported by the Ministry of Science and Higher Education of Poland (Research Project No. S/200).
Open Access This article is distributed under the terms of the Creative Commons Attribution 4.0 International License (http:// creativecommons.org/licenses/by/4.0/), which permits unrestricted use, distribution, and reproduction in any medium, provided you give appropriate credit to the original author(s) and the source, provide a link to the Creative Commons license, and indicate if changes were made.

\section{References}

1. Abrantes J, van der Loo W, Le Pendu J, Esteves PJ (2012) Rabbit haemorrhagic disease (RHD) and rabbit haemorrhagic disease virus (RHDV): a review. Vet Res 43:12. doi:10.1186/1297-9716-43-12

2. Altschul SF, Gish W, Miller W, Myers EW, Lipman DJ (1990) Basic local alignment search tool. J Mol Biol 215:403-410

3. Jl Baily, Dagleish MP, Graham M, Maley M, Rocchi MS (2014) RHDV variant 2 persistence detected in Scotland. Vet Rec 19:174-190

4. Capucci L, Fusi P, Lavazza A, Pacciarini ML, Rosi C (1996) Detection and preliminary characterization of a new rabbit calicivirus related to haemorrhagic disease virus but nonpathogenic. J Virol 70:8614-8623

5. Capucci L, Fallacara F, Grazioli S, Lavazza A, Pacciarini ML, Brocchi E (1998) A further step in the evolution of rabbit haemorrhagic disease virus: the appearance of the first consistent antigenic variant. Virus Res 58:115-126

6. Chrobocińska M, Mizak B (2007) Phylogenetic analysis of partial capsid protein gene of rabbit haemorrhagic disease virus (RHDV) strains isolated between 1993 and 2005 in Poland. Bull Vet Inst Pulawy 51:189-197

7. Dalton KP, Nicieza I, Balseiro A, Muguerza MA, Rosell JM, Casais R, Alvarez AL, Parra F (2012) Variant haemorrhagic disease virus in young rabbits, Spain. Emerg Infect Dis 18:2009-2012

8. Esteves PJ, Abrantes J, Bertagnoli S, Cavadini P, Gavier-Widen D, Guitton JS, Lavazza A, Lemaitre E, Letty J, Lopes AM, Neimanis A, Ruvoen-Clouet N, Le Pendu J, Marchandeau S, Le GallRecule G (2015) Emergence of pathogenicity in Lagoviruses: evolution from pre-existing non-pathogenic strains or through a species jump? PLOS Pathog. doi:10.1371/journal.ppat.1005087

9. Fitzner A (2009) Characterization and immunogenic properties of Polish strains of RHD virus. Bull Vet Inst Pulawy 53:575-582

10. Fitzner A, Kęsy A (2003) Variability of Polish isolates of the RHD virus. Med Weter Wet. 59:905-908 (in Polish)

11. Fitzner A, Niedbalski W, Paprocka G, Kęsy A (2012) Identification of Polish RHDVa subtype strains based on the analysis of a highly variable part of VP60 gene. PJVS 15:21-29

12. Fitzner A, Niedbalski W (2016) Serological survey for RHD antibodies in rabbits from two types of rabbit breeding farms. PJVS 19:597-607

13. Gorski J, Mizak B, Mizak Z, Komorowski A (1988) Clinical and anatomopathological features of rabbits peste (viral haemorrhagic disease of rabbits). Życie Weterynaryjne 63:266-269 (in Polish)

14. Kęsy A, Fitzner A, Niedbalski W, Paprocka G, Walkowiak B (1996) A new variant of the viral haemorrhagic disease of rabbits virus. Rev Sci Tech 15:1029-1035

15. Le Gall G, Huguet S, Vende P, Vautherot JF, Rasschaert D (1996) European brown hare syndrome virus: molecular cloning and sequencing of the genome. J Gen Virol 77:1693-1697

16. Le Gall-Recule G, Zwilgenstaein F, Laurent S, de Boisseson C, Portejoie Y, Rasschaert D (2003) Phylogenetic analysis of rabbit 
haemorrhagic disease virus in France between 1993 and 2000, and the characterization of RHDV antigenic variants. Arch Virol 148:65-81

17. Le Gall-Recule G, Zwilgenstaein F, Boucher S, Le Normand B, Plassiart G, Portejoie Y, Decors A, Bertagnoli S, Guerin JL, Marchandeau S (2011) Detection of a new variant of haemorrhagic disease virus in France. Vet Rec 5:137-138

18. Le Gall-Recule G, Lavazza A, Marchandeau S, Bertagnoli S, Zwilgenstaein F, Cavadini P, Martinelli N, Lombardi G, Guerin JL, Lemaitre E, Décors A, Boucher S, Le Normand B, Capucci L (2013) Emergence of a new lagovirus related to rabbit haemorrhagic disease virus. Vet Res 44:81

19. Liu SJ, Xue HP, Pu BQ, Quian NH (1984) A new viral disease in rabbits. Anim Husb Vet Med 16:253-255

20. Lopes AM, Marques S, Silva E, Magalhaes MJ, Pinheiro A, Alves PC, Le Pendu J, Esteves PJ, Thomson G, Abrantes J (2014) Detection of RHDV strains in the Iberian hare (lepus granatensis): earliest evidence of rabbit labovirus cross-species infection. Vet Res 45:94

21. Lopes AM, Dalton KP, Magalhaes MJ, Parra F, Esteves PJ, Holmes EC, Abrantes J (2015) Full genomic analysis of new variant rabbit hemorrhagic disease virus revealed multiple recombination events. J Gen Virol 96:1309-1319

22. Luttermann C, Meyers G (2010) Feline calicivirus in caliciviruses molecular and cellular virology. In: Hansman GS, Jiang
XJ, Green KY (eds) Chapter 8. Caister Academic Press, Norfolk, pp 145-166

23. Meyers GC, Wirblich C, Thiel HJ (1991) Rabbit haemorrhagic disease virus molecular cloning and nucleotide sequencing of a calicivirus genome. Virology 184:664-676

24. Meyers GC, Wirblich C, Thiel HJ (1991) Genomic and subgenomic RNAs of rabbit hemorrhagic disease virus are both protein-linked and packaged into particles. Virology 184:677-686

25. Meyers G, Wirblich C, Thiel HJ, Thumfart JO (2000) Rabbit haemorrhagic disease virus: genome organization and polyprotein processing of calicivirus studied after transient expression of cDNA constructs. Virology 276:349-363

26. Moss SR, Turner SL, Trout RC, White PJ, Hudson PJ, Desai A, Armesto M, Forrester NL, Gould EA (2002) Molecular epidemiology of rabbit haemorrhagic disease virus. J Gen Virol 83:2461-2467

27. Schirrmeirer H, Reimann I, Kollner B, Granzow H (1999) Pathogenic, antigenic and molecular properties of rabbit haemorrhagic disease virus (RHDV) isolated from vaccinated rabbits: detection and characterization of antigenic variants. Arch Virol 144:719-735

28. Tamura K, Stecher G, Peterson D, Filipski A, Kumar S (2013) MEGA6: Molecular Evolutionary Genetics Analysis version 6.0. Mol Biol Evol 30:2725-2729 